\title{
Dispersive Controlling of Femtosecond Laser Radiation: New Opportunities and Developments
}

\author{
V.Ya. Molchanov* And K.B. YushKov \\ Acousto-Optical Research Center, National University of Science and Technology "MISIS", \\ 4, Leninsky prospekt, 119049 Moscow, Russia
}

\begin{abstract}
The paper is devoted to new trends in dispersive control techniques for ultrafast light emission. Acoustooptical dispersive delay lines for controlling the spectral components and phase composition of ultrashort laser pulses are considered. The method of super high frequency modulation of chirped femtosecond laser pulses is proposed. Theoretical approach to dispersive pulse shaping is supported by the experiments.
\end{abstract}

DOI: 10.12693/APhysPolA.127.20

PACS: $42.60 . \mathrm{By}, 42.79 . \mathrm{Jq}$

\section{Introduction}

Currently, high power femtosecond laser systems can produce extremely high intensities required for many applications in physics, chemistry, medicine, industry etc. $[1,2]$. Wide range of applications require ultrashort Fourier-transform-limited (FTL) flat-phase pulses without pedestals or pre-pulses and having high contrast ratio $[2,3]$.

Typical architecture of a high-power laser system consists of a master oscillator, a stretcher, an amplification chain, and a compressor. Even though femtosecond master oscillators usually generate FTL laser pulses, it is practically impossible to obtain pulses with flat spectral phase after amplification and compression. All the elements of the optical path possess higher-order optical dispersions and spectral dependence of throughput that leads to the spectral phase violation of seed femtosecond pulses and amplitude spectral distortions in the optical amplifiers [2-4]. The stretcher-compressor pairs are designed to compensate for the group delay dispersion, however controlling of higher-order dispersions is hardly possible [5].

The most efficient solution to the problem of spectral phase and amplitude distortions is to insert an adaptive dispersive optical device in the optical path of the laser system. That device should perform precompensation for higher-order dispersions and spectrum distortion in the laser system by controlling the spectral amplitude and the spectral phase of the seed femtosecond pulses.

Currently, three methods for controlling the spectral phase are known: chirped mirrors, grating-based spectral domain shapers with liquid crystal spatial light modulators, and adaptive dispersive delay lines based on the acoustooptical effect. The experience of employment of powerful laser systems shows that the acoustooptical method has a number of incontestable advantages, such as continuous phase control, independent controlling of

${ }^{*}$ corresponding author the spectral phase and the spectral amplitude, high-speed adaptability of transmission parameters. Owing to these advantages, acoustooptical dispersive delay lines proved to be the best among such devices.

For the first time the idea of light pulse compression by means of non-collinear Bragg diffraction was fragmentarily considered by McMahon in 1967 [6]. Pozhar and Pustovoit proposed application of the acoustooptical collinear interaction for compression of ultrashort laser pulses in 1986 [7].

According to the original concept developed in Ref. [7], the birefringence of the crystal in which acoustooptical interaction takes place is used for pulse compression. The input beam is polarized along the fast axis of the crystal light velocity. Collinear acoustooptical interaction converts each spectral component from the fast mode into the slow mode with orthogonal polarization (Fig. 1). The amplitude and the phase of each spectral component at the output of the crystal are determined by the ultrasonic spectral amplitude and by the spatial position of the corresponding ultrasound frequency in the interaction region. Thus long-wavelength components are delayed relatively to the short-wavelength components of emission.

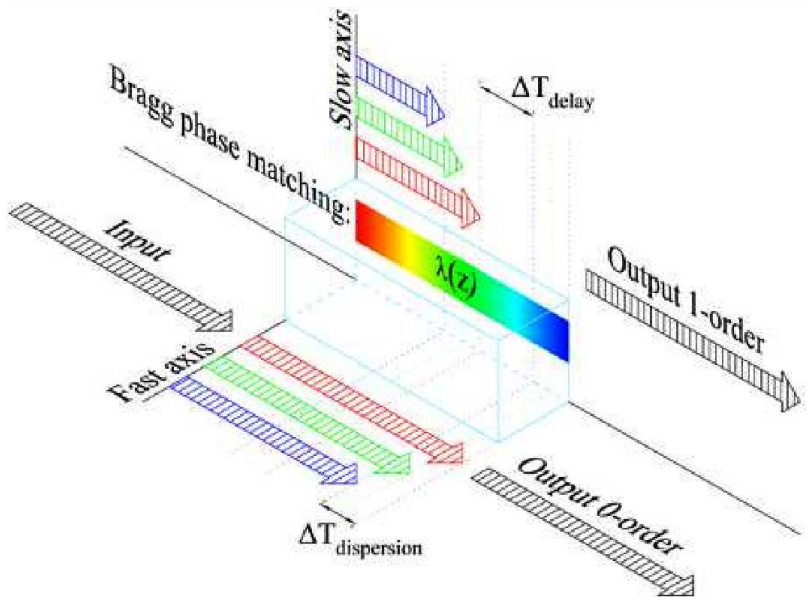

Fig. 1. Dispersive delay line model. 
Fermann et al. used that concept for spectral shaping of laser pulses [8]. Tournois developed that method for advanced amplitude and phase control of femtosecond laser radiation $[9,10]$. Presently, this acoustooptical technique is widely used in femtosecond systems. The further development of dispersive technique was made by Molchanov [11] for CPA and OCPCA laser systems. Nowadays the dispersive acoustooptical methods are demanded in design of femtosecond fiber lasers [12] and optical amplifiers [11], adaptive spectroscopy, telecommunications [13], etc.

This paper is devoted to new trends in the field of acoustooptical dispersive control of the light emission. Suppression of spectral transmission function side-lobes is studied as well as spectral coding of broadband radiation. The method of acoustooptical super high frequency (SHF) modulation of the chirped femtosecond laser pulses is proposed, dispersive equalization of the optical amplifier gain is considered. Experimental results obtained with different femtosecond laser systems are presented.

\section{Quasi-LFM dispersive method} of the synthesis of complex spectral transmission

Definition of higher-order dispersions (HOD) is widely used in laser technique. The spectral phase $\Phi(\omega)$ of the optical wave can be expanded in a Taylor series in the vicinity of central optical frequency $\omega_{0}$ :

$$
\begin{aligned}
& \Phi(\omega)=\Phi\left(\omega_{0}\right)+\Phi^{\prime}\left(\omega_{0}\right)\left(\omega-\omega_{0}\right) \\
& \quad+\frac{1}{2} \Phi^{\prime \prime}\left(\omega_{0}\right)\left(\omega-\omega_{0}\right)^{2}+\ldots= \\
& \Phi\left(\omega_{0}\right)+D_{1}\left(\omega-\omega_{0}\right)+D_{2}\left(\omega-\omega_{0}\right)^{2}+\ldots
\end{aligned}
$$

The polynomial coefficients $D_{n}$ are named the dispersions of the $n$-th order. The first order dispersion $D_{1}$ determines a time delay of the wave packet. The second order dispersion $D_{2}$ which is often referred to as "group velocity dispersion" may be caused by different factors, for example, by acoustooptical diffraction by linear frequency modulation (LFM) sound wave, by internal crystal dispersion, etc.

It can be shown that in the case of LFM signal with the frequency chirp $\Delta F$ and Gaussian envelope having the width of $T_{0}$ the induced second-order dispersion $D_{2}$ is expressed as follows [14]:

$$
D_{2} \approx \pi T_{0} / \beta^{2} \Delta F \text {. }
$$

Here $\beta$ is the ratio of the light frequency to the ultrasound frequency defined by the wave vector diagram of acoustooptical interaction.

Pure LFM of ultrasound for transmission function synthesis was first studied by Magdich et al. in 1980 [15, 16]. The amplitude of the ultrasonic wave was constant. From the modern point of view, that method is equivalent to the inducing only the second-order dispersion $D_{2}$. Higher-order dispersions $D_{3}, D_{4} \ldots D_{n}$ were assumed to be equal to zero.
Molchanov and Yushkov in series of the papers proposed complex transmission function synthesis based on quasi-LFM of acoustic waves including non-zero higher order dispersions $D_{3}, D_{4} \ldots D_{n}[14,17-19]$. That method consists of the following. The spectral transmission function of acoustooptical filter $T(\omega)$ is determined by the Fourier transform of complex sound field distribution along the crystal $S(t)=A(t) \exp (\mathrm{i} 2 \pi f(t) t)$,

$$
T(\omega) \sim \int_{0}^{\frac{L}{V}} S(t) \exp (\mathrm{i} 2 \pi \omega t) \mathrm{d} t,
$$

where $A(t)$ is the temporal amplitude sound distribution, $f(t)$ is the temporal frequency (phase) distribution of ultrasound.

Using the inverse Fourier transform, the complex ultrasonic waveform $S(t)$ is calculated providing the desirable transmission function $T(\omega)$. The main idea for transmission function synthesis is using quasi-LFM ultrasonic waveforms instead of pure LFM i.e. LFM accompanied with phase and amplitude modulation. Generally, the spectra of incoming light, of ultrasound in the acoustooptical crystal, and of diffracted light are described by the complex functions.

According to this approach, sophisticated adaptive spectral apparatus for arbitrary transmission function dispersive synthesis was designed and fabricated (Fig. 2). The hardware system includes quasi-collinear acoustooptical devices based on the paratellurite single crystals and electronic control system. The corresponding algorithms and software were also created. The user should simply set the arbitrary profile of the spectral transmission function.

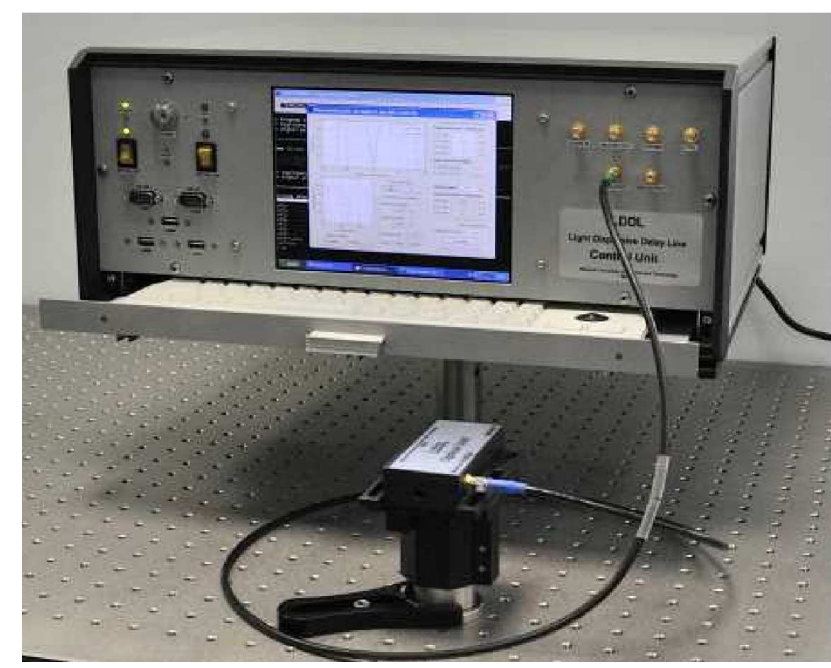

Fig. 2. Original apparatus for dispersive light control.

\section{Transmission function side-lobes suppression and multichannel spectral binary coding}

It is well known that suppression of the side-lobes of the transmission function of the acoustooptical filter by 
means of ultrasound field apodization better than 18$20 \mathrm{~dB}$ is a rather complicated problem in practice. That limits the spectral contrast of the acoustooptical devices. The ultrasound field synthesis in the complex domain seems to be efficient instrumental tool for side-lobes suppression and for increase of spectral contrast. For example, side-lobes suppression of the rectangular transmission function can reach more than $24 \mathrm{~dB}$ (Fig. 3). Moreover, the spectral transmission function shape is very close to the rectangular one having high spectrum rise value more than $26 \mathrm{~dB} / \mathrm{nm}$. The spectral width of transmission function at $-3 \mathrm{~dB}$ level is $2 \mathrm{~nm}$. The intrinsic spectral width of quasi-collinear acoustooptical filter used in the experiment is $0.16 \mathrm{~nm}$ at $800 \mathrm{~nm}$ in the single frequency regime. The measurements were made using femtosecond master oscillator with 11 fs FTL pulse duration (Femtosource Synergy by Femtolasers $\mathrm{GmbH}$ ).

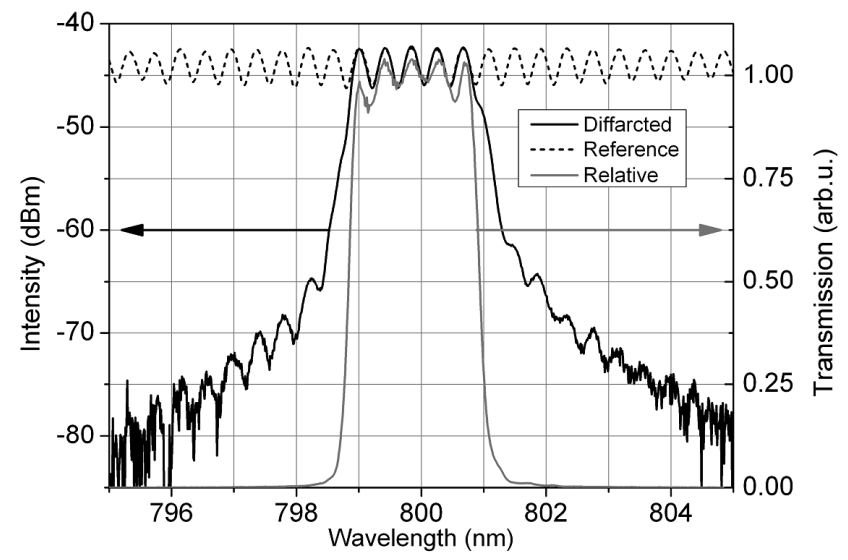

Fig. 3. Rectangular transmission window (black curve; left scale) and side-lobes suppression of the transmission function (gray curve; right scale).

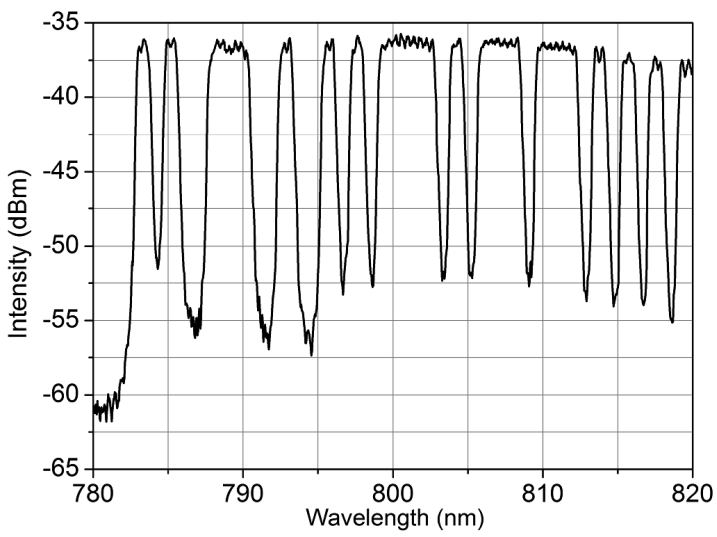

Fig. 4. The fragment of 64-channel spectral coding of a broadband $(\Delta \lambda=120 \mathrm{~nm}$ ) laser emission.

The example of the spectral encoding of broadband femtosecond laser radiation (spectral bandwidth $\Delta \lambda=120 \mathrm{~nm}$ FWHM) with a pseudorandom binary sequence (PRBS) is illustrated in Fig. 4. Spectral width of each channel corresponding to one encoded bit is $1 \mathrm{~nm}$ at $-3 \mathrm{~dB}$ level, channel-to-channel contrast is better than $-18 \mathrm{~dB}$ when measured similarly to spectral modulation transfer function [20]. The total number of independently encoded channels in PRBS is 64 .

\section{Gigahertz modulation}

of the chirped femtosecond laser radiation by dispersive acoustooptical method

It is well-known that modulation bandwidth (rise time) of acoustooptical devices is defined by the travel time of ultrasonic wave through the interaction region in the crystal. The dispersive controlling can overcome that limitation. The reason is that dispersive delay line provides direct parallel light-to-light spectral conversion, while sound field space distribution can be considered as frozen. For chirped laser pulses that results in time-domain modulation with the frequencies much higher than the modulation bandwidth of the ultrasonic waveform. The experimental setup is presented

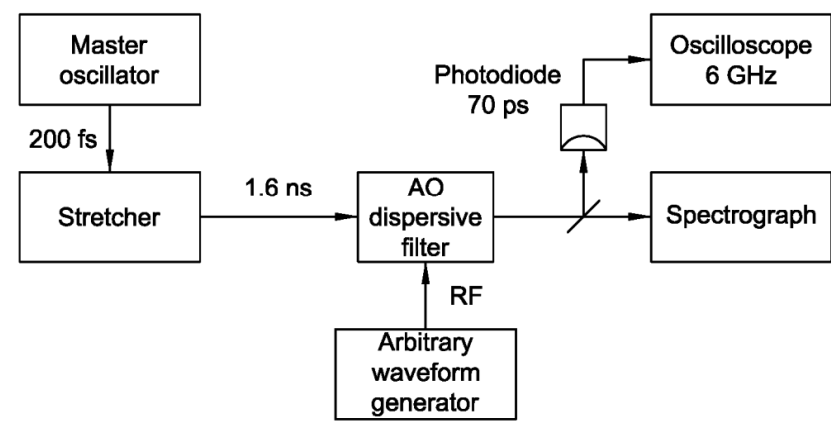

Fig. 5. Scheme of the experimental setup for SHF modulation.

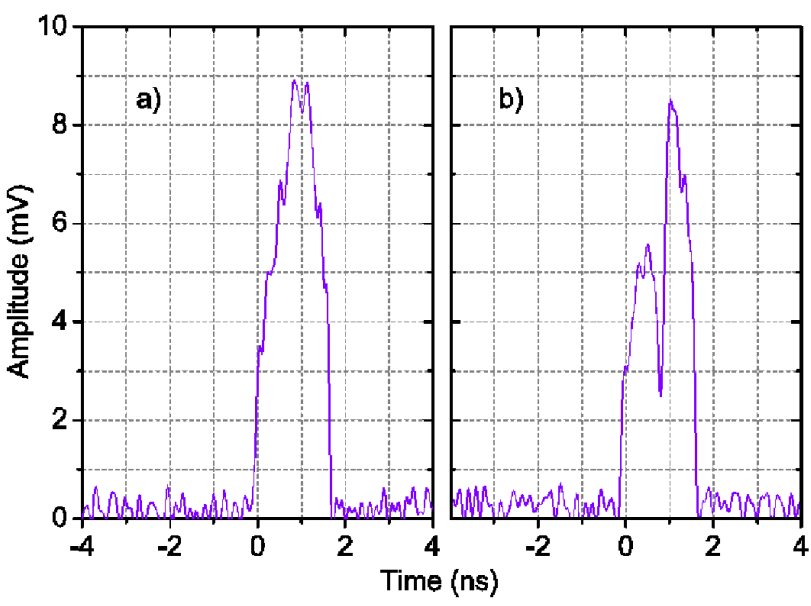

Fig. 6. The temporal profile of the chirped laser pulse (a) incoming; (b) pulse-modulated $\left(t_{\text {rise }}<100 \mathrm{ps}\right)$.

in Fig. 5. The experiments were performed in a laser system based on Nd-doped phosphate glass. The master oscillator generates FTL ultrashort pulses of $200 \mathrm{fs}$ duration $(\lambda=1054 \mathrm{~nm}, \Delta \lambda=8 \mathrm{~nm})$. After the stretcher, the chirped pulses had FWHM duration $1.6 \mathrm{~ns}$, while 
the bandwidth was equal to $\Delta \lambda=4 \mathrm{~nm}$. Quasicollinear acoustooptical filter based on paratellurite was placed in the optical path after the stretcher. Transmission function of the filter was $0.12 \mathrm{~nm}$ FWHM at the wavelength $1054 \mathrm{~nm}$ in a single frequency regime. Fast photodiodes (pulse response less than $70 \mathrm{ps)} \mathrm{were} \mathrm{used} \mathrm{in} \mathrm{the} \mathrm{exper-}$ iment with the $6 \mathrm{GHz}$ bandwidth oscilloscope. The incoming temporal shape of a chirped laser pulse is presented in Fig. 6a. The dispersive modulation of ultrasonic wave using RF arbitrary waveform generator provided narrow rejection band in the spectrum, resulting in correspondent time-domain modulation of the laser pulse (Fig. 6b). The dip width was 175 ps corresponding to the spectral bandwidth of $0.7 \mathrm{~nm}$, the estimated rise time less than $90 \mathrm{ps}$. Shorter rise time could not be measured correctly because of the limited time response (more than $100 \mathrm{ps)}$ ) of the registration system [21]. In other words, dispersive controlling can provide modulation bandwidth of the pulsed laser radiation higher than $10 \mathrm{GHz}$, i.e. in SHF domain.

\section{Dispersive equalization of the optical regenerative amplifier spectral gain}

The achieved advances in software and instrumentation development were applied for creation of the dispersive acoustooptical equalizer of the spectral gain in a regenerative amplifier on the base of Nd-doped phosphate glass [21]. Without equalization, narrowing of the emission spectrum in the regenerative amplifier with unsaturated gain took place mainly during the first several passes of the pulse through the active medium. Acoustooptical spectral equalizer having outstanding resolution of the order $\lambda / \delta \lambda \approx 10^{4}$ at $1054 \mathrm{~nm}$ was fabricated to provide the spectral control of amplifier gain in the bandwidth of $\Delta \lambda=10 \mathrm{~nm}$ during every optical pass.

The master oscillator, stretcher (before amplification chain) and the measurement system after the ampli-

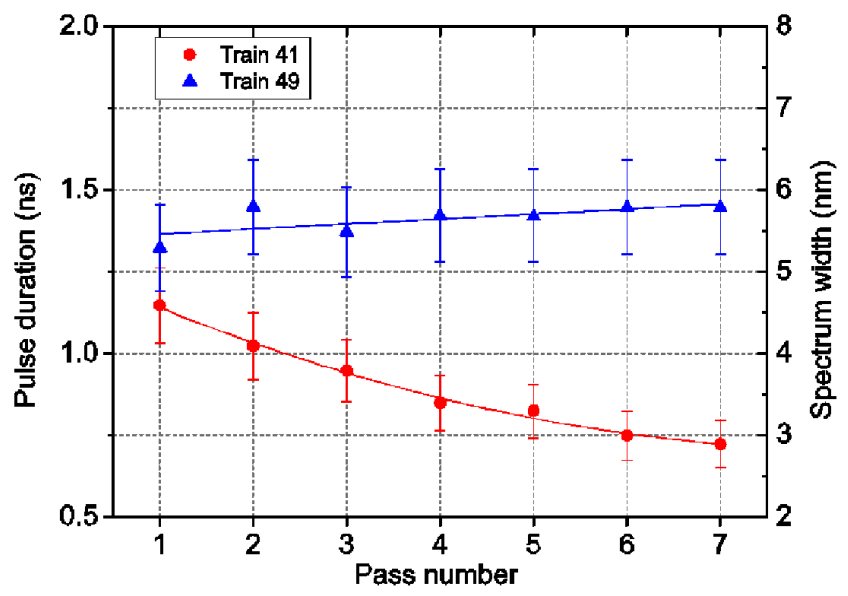

Fig. 7. The chirped laser pulse spectral width (duration) vs. no. of round trips in the regenerative amplifier. Upper curve: equalizer is on, lower curve: equalizer is off. fier were the same as shown in Fig. 5. Equalizer was placed inside the cavity of the regenerative amplifier. It was advisable to use the zero-order beam at the output of the equalizer while the Bragg output was additionally used for controlling emission parameters. The experimental results for the first 7 round trips are presented in Fig. 7. In a regular mode of operation without spectral gain control (Fig. 7a) a steady reduction of the chirped laser pulse spectrum (duration) took place. When the equalizer was turned on, the spectral dip with the width of $3.5 \mathrm{~nm}$ was formed in the zero order by the dispersive method. When acoustooptical equalizer was switched on, the pulse spectrum (duration) remained practically unchanged (Fig. 7b).

\section{Conclusion}

The work presents some new opportunities and trends of adaptive dispersive controlling of ultrashort laser radiation. Adaptive dispersive synthesis of complex spectral transmission functions by means of acoustooptics is considered. Acoustooptical devices, electronic control system, mathematical algorithms, and corresponding software were created. Spectral encoding of broadband $(\Delta \lambda=120 \mathrm{~nm}$ ) radiation was demonstrated for $64-$ bit pseudo-random binary sequence. The novel method of adaptive acoustooptical modulation of chirped laser pulses in super high frequency domain (bandwidth more than $10 \mathrm{GHz}$ ) was proposed and demonstrated experimentally. Acoustooptical spectral equalizer was created for gain control in the $\mathrm{Nd}$-doped phosphate glass optical regenerative amplifier with the bandwidth $\Delta \lambda=$ $10 \mathrm{~nm}$. The obtained results will serve as material for further research.

\section{Acknowledgments}

The work was carried out with financial support in part from the Ministry of Education and Science of the Russian Federation in the framework of Increase Competitiveness Program of NUST "MISiS" (No. K1-2014008) and in project No. 3035023 and from Russian Foundation for Basic Research in projects No. 12-07-00641, No. 12-07-00642.

\section{References}

[1] A.A. Shaykin, G.I. Freidman, S.G. Garanin, V.N. Ginzburg, E.V. Katin, A.I. Kedrov, E.A. Khazanov, A.V. Kirsanov, V.V. Lozhkarev, G.A. Luchinin, L.V. L'vov, A.N. Mal'shakov, M.A. Martyanov, V.A. Osin, O.V. Palashov, A.K. Poteomkin, N.N. Rukavishnikov, V.V. Romanov, A.V. Savkin, A.M. Sergeev, S.A. Sukharev, O.V. Trikanova, I.N. Voronich, I.V. Yakovlev, B.G. Zimalin, in: CLEO/Europe and EQEC 2009 Conference Digest, Eds.: R. de la Rue, J. Dudley, D. Meschede, K. Moelmer, P. Pinske, IEEE, Munich 2009, p. 1.

[2] A.V. Korzhimanov, A.A. Gonoskov, E.A. Khazanov, A.M. Sergeev, Phys.-Usp. 54, 9 (2011).

[3] A.M. Weiner, Ultrafast Optics, Wiley, New York 2009. 
[4] V.V. Lozhkarev, G.I. Freidman, V.N. Ginzburg, E.V. Katin, E.A. Khazanov, A.V. Kirsanov, G.A. Luchinin, A.N. Mal'shakov, M.A. Martyanov, O.V. Palashov, A.K. Poteomkin, A.M. Sergeev, A.A. Shaykin, I.V. Yakovlev, Laser Phys. Lett. 4, 421 (2007).

[5] I.V. Yakovlev, Quantum Electron. 44, 393 (2014).

[6] D.H. McMahon, Proc. IEEE 55, 1602 (1967).

[7] V.E. Pozhar, V.I. Pustovoit, Sov. J. Quantum Electron. 17, 509 (1987).

[8] M.E. Fermann, V. da Silva, D.A. Smith, Y. Silberberg, A.M. Weiner, Opt. Lett. 18, 1505 (1993).

[9] P. Tournois, Opt. Commun. 140, 245 (1997).

[10] T. Oksenhendler, D. Kaplan, P. Tournois, G.M. Greetham, F. Estable, Appl. Phys. BLasers Opt. 83, 491 (2006).

[11] V.Ya. Molchanov, S.I. Chizhikov, O.Yu. Makarov, N.P. Solodovnikov, V.N. Ginzburg, E.V. Katin, E.A. Khazanov, V.V. Lozhkarev, I.V. Yakovlev, Appl. Opt. 48, C118 (2009).

[12] R. Royon, J. Lhermite, L. Sarger, E. Cormier, Opt. Express 21, 13818 (2013).

[13] V.V. Proklov, O.A. Byshevski-Konopko, V.I. Grigorievski, Quantum Electron. 43, 542 (2013).
[14] V. Molchanov, S. Chizhikov, K. Yushkov, Quantum Electron. 41, 675 (2011).

[15] L.N. Magdich, Opt. Spectrosc. 49, 387 (1980).

[16] L.N. Magdich, V.Ya. Molchanov, I.P. Ponomareva, Opt. Spectrosc. 56, 736 (1984).

[17] V.Ya. Molchanov, K.B. Yushkov, AIP Conf. Proc 1433, 72 (2012).

[18] V.Ya. Molchanov, S.I. Chizhikov, K.B. Yushkov, in: Proc. Ultrafast Optics 2011, Monterey (CA), Lawrence Livermore National Laboratory, Monterey (CA) 2011, p. 85 .

[19] K. Yushkov, V. Molchanov, S. Chizhikov, in: Proc. Acoustics 2012 Nantes, Nantes (France), Societé Français d'Acoustique, Societé Français d'Acoustique, Nantes 2012, p. 143.

[20] K.B. Yushkov, V.Ya. Molchanov, Opt. Lett. 38, 3578 (2013).

[21] S.I. Chizhikov, S.G. Garanin, L.V. Goryachev, V.Ya. Molchanov, V.V. Romanov, N.N. Rukavishnikov, S.V. Sokolovskii, I.N. Voronich, K.B. Yushkov, Laser Phys. Lett. 10, 015301 (2013). 